\title{
LETTER \\ Measurement-Based Performance Evaluation of Coded MIMO-OFDM Spatial Multiplexing with MMSE Spatial Filtering in an Indoor Line-of-Sight Environment
}

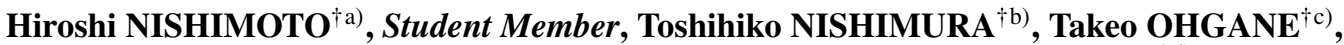 \\ and Yasutaka OGAWA ${ }^{\dagger \mathrm{d})}$, Members
}

SUMMARY The MIMO system can meet the growing demand for higher capacity in wireless communication fields. So far, the authors have reported that, based on channel measurements, uncoded performance of narrowband MIMO spatial multiplexing in indoor line-of-sight (LOS) environments generally outperforms that in non-LOS (NLOS) ones under the same transmit power condition. In space-frequency coded MIMO-OFDM spatial multiplexing, however, we cannot expect high space-frequency diversity gain in LOS environments because of high fading correlations and low frequency selectivity of channels so that the performance may degrade unlike uncoded cases. In this letter, we present the practical performance of coded MIMO-OFDM spatial multiplexing based on indoor channel measurements. The results show that an LOS environment tends to provide lower space-frequency diversity effect whereas the MIMO-OFDM spatial multiplexing performance is still better in the environment compared with an NLOS environment.

key words: MIMO, OFDM, spatial multiplexing, MMSE spatial filtering, indoor channel measurement, line-of-sight environments, space-frequency diversity

\section{Introduction}

The multiple-input multiple-output (MIMO) system, which is equipped with multiple transmit (TX) and receive (RX) antennas, can meet the growing demand for higher capacity in wireless communication fields [1], [2]. Therefore, it has been widely investigated, and some standardization groups have planned to incorporate it or have already determined to do so [3]-[5]. So far, the authors have conducted MIMO channel measurement campaigns and studied the performance of narrowband MIMO spatial multiplexing in indoor environments [6]-[8]. It was found that the uncoded performance of spatial multiplexing in line-of-sight (LOS) environments generally outperforms that in non-LOS (NLOS) ones under the same transmit power condition even though the existence of direct wave increases fading correlations in LOS environments.

Applying the orthogonal frequency division multiplexing (OFDM) technique to the MIMO system (MIMO-

Manuscript received April 16, 2007.

Manuscript revised November 25, 2007.

${ }^{\dagger}$ The authors are with the Graduate School of Information Science and Technology, Hokkaido University, Sapporo-shi, 0600814 Japan.

a)E-mail: hn@ist.hokudai.ac.jp

b)E-mail: nishim@ist.hokudai.ac.jp

c) E-mail: ohgane@ist.hokudai.ac.jp

d) E-mail: ogawa@ist.hokudai.ac.jp

DOI: 10.1093/ietcom/e91-b.5.1648
OFDM) is a practical approach because OFDM is robust to multipath fading [3]-[5]. In MIMO-OFDM spatial multiplexing, a transmitted data sequence can obtain spacefrequency diversity effect by being coded and interleaved over substreams and subcarriers. As mentioned above, the presence of the LOS component causes high fading correlations, which result in low spatial diversity gain. In addition, frequency selectivity in LOS environments tends to be lower due to such a high level component. Hence, we cannot expect high space-frequency diversity gain in LOS environments, especially when using a practical linear demultiplexing scheme such as minimum mean square error (MMSE) spatial filtering, therefore the performance may degrade unlike uncoded cases.

In this letter, we present the practical performance of coded $2 \times 2$ and $4 \times 4$ MIMO-OFDM spatial multiplexing with MMSE spatial filtering based on indoor channel measurements.

\section{Measurement Setup}

The measurement was carried out in a conference room in a building of the Graduate School of Information Science and Technology at Hokkaido University (Fig. 1). The walls of the room consisted of plasterboard around reinforced concrete pillars and metal doors. In the room, we set up TX and $\mathrm{RX}$ tables with $4 \mathrm{~m}$ separation and a vector network analyzer

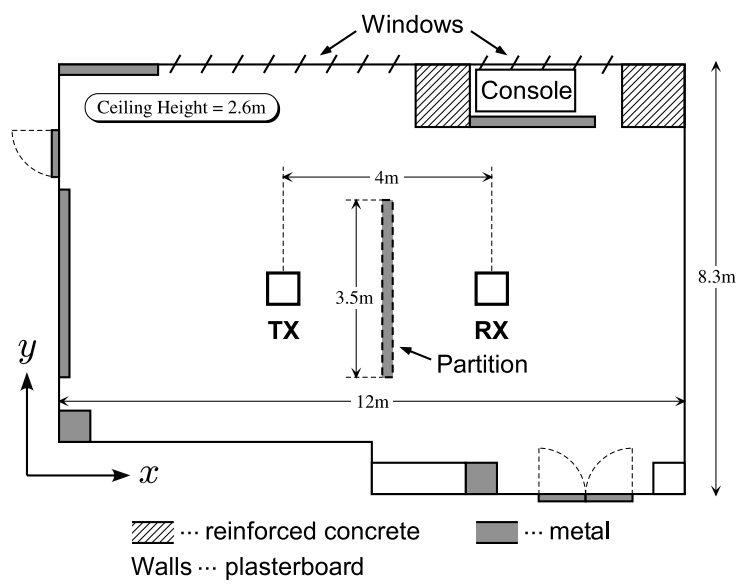

Fig. 1 Measurement site (top view). 

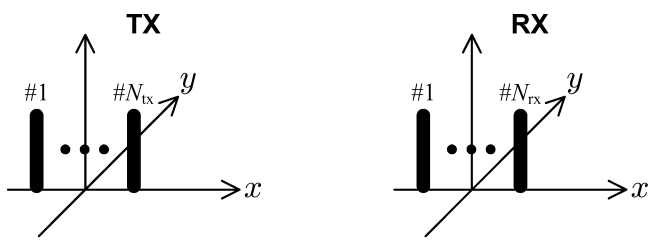

(a) $\mathrm{TX}-x / \mathrm{RX}-x$
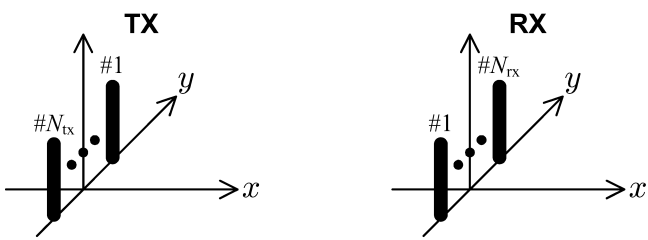

(b) $\mathrm{TX}-y / \mathrm{RX}-y$

Fig. 2 Array orientations.

to measure the channel responses. The measurement band was from $5.15 \mathrm{GHz}$ to $5.4 \mathrm{GHz}$ (bandwidth of $250 \mathrm{MHz}$ ), and it was swept with a $156.25 \mathrm{kHz}$ interval $(1,601$ frequency points). The obtained channel at each frequency point was averaged over 10 snapshots in order to reduce thermal noise included in tentative measurements. We used uniform linear arrays (ULAs) composed of omnidirectional collinear antennas as both TX and RX arrays. We examined two array orientations "TX- $x / \mathrm{RX}-x$ " and "TX- $y / \mathrm{RX}-y$ " as shown in Fig. 2, where $N_{\mathrm{tx}}$ and $N_{\mathrm{rx}}$ indicate numbers of TX and RX antennas, respectively. Two antenna spacing (AS) scenarios $\lambda / 2$ and $1 \lambda$ were considered, where $\lambda$ denotes the wavelength at $5 \mathrm{GHz}$ (i.e., $\lambda=6 \mathrm{~cm}$ ). In the LOS condition there was no obstructing object between the TX and RX tables, and in the NLOS condition a metal partition was located between them. The estimated $K$-factor in the LOS condition was $1.66 \mathrm{~dB}$ in single-antenna measurement without mutual coupling effect [8]. By changing TX and RX array positions, we obtained $7 \times 7=49$ spatially different data while keeping the distance of $4 \mathrm{~m}$ between the TX and RX tables, where an interval between adjacent array positions was $\lambda / 4(1.5 \mathrm{~cm})$ [7]-[9]. Channel data were obtained while no one was in the room to ensure statistical stationarity of propagation. Details on the measurement setup are presented in [6]-[9]. Also, details on the $K$-factor estimation and characteristics of the measured channel data, e.g., CDFs of channel amplitudes, etc., are reported mainly in [8].

\section{Numerical Analysis}

\subsection{Simulation Conditions}

Using measured channel data, we examined the bit error rate (BER) performance of spatially-multiplexed $2 \times 2$ and $4 \times 4$ MIMO-OFDM systems by computer simulations. Some of the simulation parameters were determined according to the contents of the IEEE802.11n standardization [3]. The transmission bandwidth was assumed to be $20 \mathrm{MHz}$,

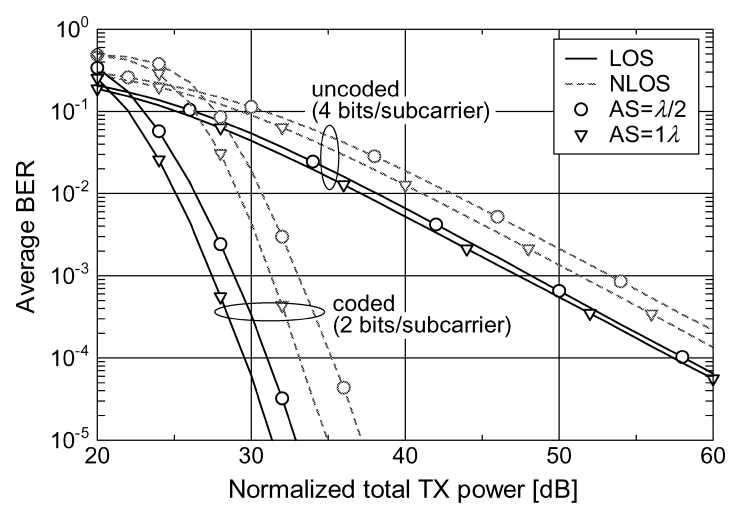

(a) $\mathrm{TX}-x / \mathrm{RX}-x$

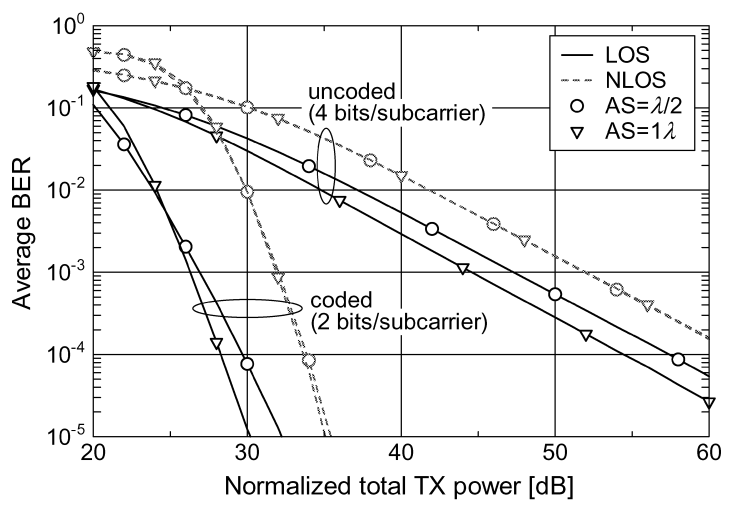

(b) $\mathrm{TX}-y / \mathrm{RX}-y$

Fig. 3 BER performance for $2 \times 2$ MIMO-OFDM.

which corresponds to 128 measurement frequency samples $(156.25 \mathrm{kHz} \times 128=20 \mathrm{MHz})$. Therefore, the total number of OFDM channel data (i.e., the number of trial frames) became $(1,601-128) \times 49=72,177$. Each set of 128-point channel data was decimated to 64 points $(312.5 \mathrm{kHz}$ interval), spectrally shaped, and normalized to the direct wave amplitude. After applying the IFFT to the modified channel data, we used its first 16 samples (guard interval duration) as the corresponding channel impulse responses. 56 subcarriers out of 64 in total were used for data transmission, and a data frame was composed of eight OFDM symbols. QPSK modulation was employed. A data sequence was coded by a convolutional encoder (constraint length of 7 and coding rate of 1/2) and then randomly bit-interleaved over substreams and subcarriers to obtain sufficient spacefrequency diversity gain. The receiver demultiplexed the received signal using an MMSE spatial filter at each subcarrier and then passed the data to a soft-decision Viterbi decoder. To sufficiently reflect space-frequency diversity effect after demultiplexing, we multiplied a log-likelihood ratio by the corresponding SINR at the MMSE spatial filter output [10]. It was assumed that a fading condition was static over a frame, and that the receiver had perfect channel state information. 


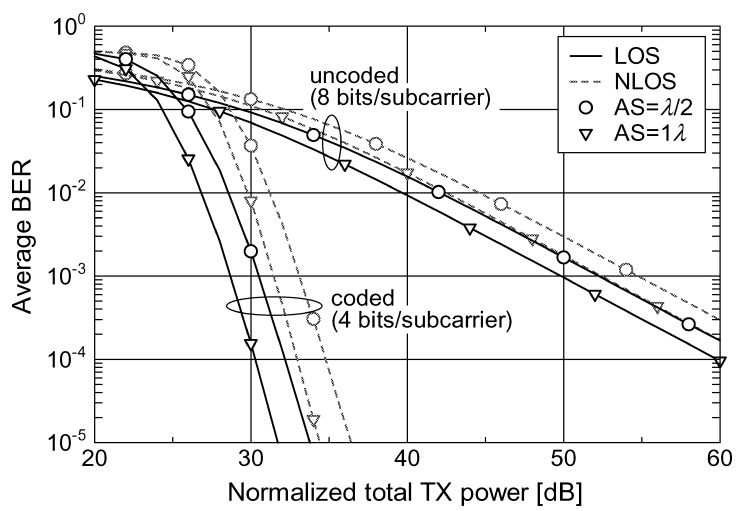

(a) $\mathrm{TX}-x / \mathrm{RX}-x$

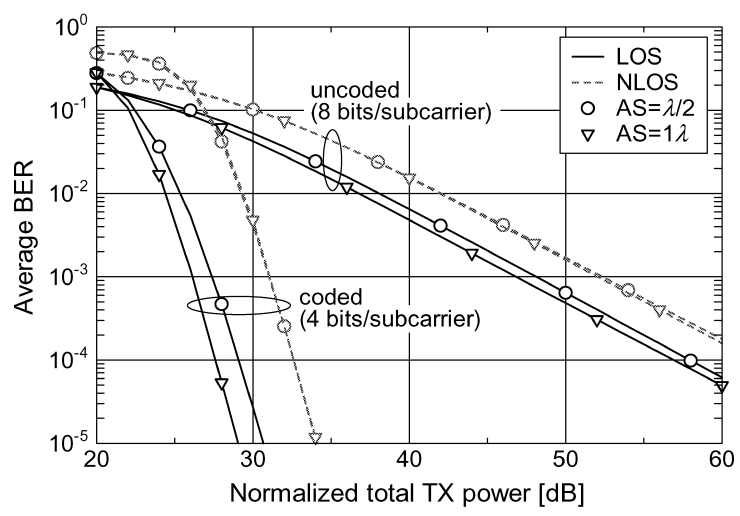

(b) $\mathrm{TX}-y / \mathrm{RX}-y$

Fig. 4 BER performance for $4 \times 4$ MIMO-OFDM.

\subsection{Simulation Results}

BER performances for $2 \times 2$ and $4 \times 4$ MIMO-OFDM are shown in Figs. 3 and 4, respectively. We also present fading correlations for all the MIMO configurations in Table 1, where only RX correlations are listed because TX and RX correlations are similar. For $4 \times 4$ MIMO cases, each listed value is averaged over six correlations. (The number of antenna pairs in a four-element array is six). Details on the correlations are discussed in [7], [8]. Here the normalized total TX power denotes the total TX power yielding average $E_{s} / N_{0}$ of $0 \mathrm{~dB}$ in the case of single-antenna OFDM transmission in an anechoic chamber with the same measurement setup. In addition to the coded case, we demonstrate the performance without coding for comparison. The uncoded case shows almost the same tendency as the narrowband case reported in [6]-[8]. From the results of the uncoded case, we can see that the LOS condition provides better BER performance compared with the NLOS condition. As reported in [6]-[8], this is because of higher received power given by the LOS component whereas it makes channels correlated as evidenced by Table 1. Also it should be noted that all the BER curves show first-order diversity regardless of the MIMO configuration.

From Table 1, we can see that the fading correlation in the case of TX- $y / \mathrm{RX}-y$ with AS $=\lambda / 2$ in the LOS condition
Table 1 RX fading correlations (averaged correlations for $4 \times 4$ MIMO).

\begin{tabular}{|c|c||c|c|c|c|}
\hline \multicolumn{2}{|c||}{} & \multicolumn{2}{c|}{ TX- $x /$ RX- $x$} & \multicolumn{2}{c|}{ TX- $y /$ RX- $y$} \\
\cline { 3 - 6 } \multicolumn{2}{|c||}{} & AS $=\lambda / 2$ & AS $=1 \lambda$ & AS $=\lambda / 2$ & AS $=1 \lambda$ \\
\hline \multirow{2}{*}{$2 \times 2$} & LOS & 0.63 & 0.54 & 0.86 & 0.47 \\
\cline { 2 - 6 } & NLOS & 0.31 & 0.10 & 0.29 & 0.04 \\
\hline \multirow{2}{*}{$4 \times 4$} & LOS & 0.41 & 0.47 & 0.82 & 0.32 \\
\cline { 2 - 6 } & NLOS & 0.23 & 0.08 & 0.21 & 0.07 \\
\hline
\end{tabular}

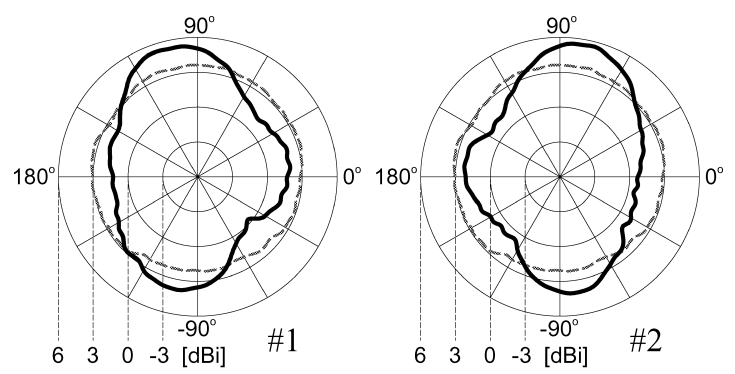

(a) $\mathrm{AS}=\lambda / 2$

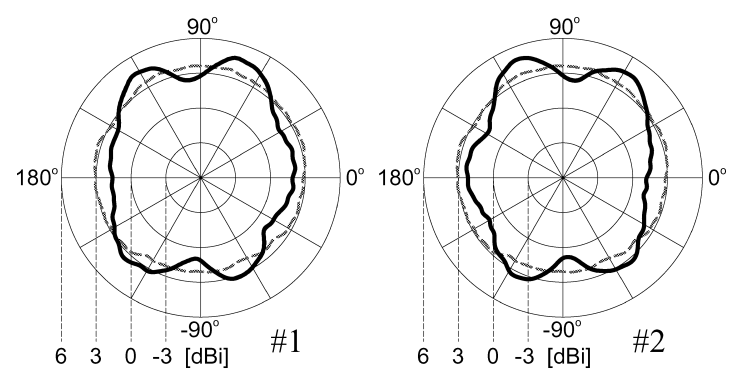

(b) $\mathrm{AS}=1 \lambda$

Fig. 5 Antenna patterns for a single antenna (dashed) and for each two-element array with mutual coupling (solid).

is significantly high in both the $2 \times 2$ and $4 \times 4$ MIMO systems. This is related not only to the narrow AS but also to mutual coupling effect. It is well known that antenna patterns are distorted by mutual coupling effect when antennas are closely arranged. Figures 5 and 6 show patterns of antennas used in the measurement for two-element arrays and four-element ULAs, respectively. For comparison, we also illustrate a pattern of a single isolated antenna in each part of these figures. The antenna index in each pattern corresponds to the one in Fig. 2. In the LOS condition, the direct wave direction in the TX- $x / \mathrm{RX}-x$ array orientation is $0^{\circ} / 180^{\circ}$, and that in the TX-y/RX- $y$ array orientation is $90^{\circ}$. Note that only in the case of TX- $y / \mathrm{RX}-y$ with AS $=\lambda / 2$, the antenna gain in the direct wave direction is higher than the single-antenna case. Therefore, the highest fading correlation in the case is due to the LOS component equivalentlyemphasized by the increased antenna gain. Such a high correlation tends to affect the diversity gain as discussed in the following.

We can evaluate the space-frequency diversity effect of a MIMO-OFDM system with the gradient of its BER curve. While the LOS condition still gives better performance than the NLOS condition even in the coded case, gradients of BER curves in LOS cases tend to be gentler than those in NLOS cases and to depend on the array configura- 


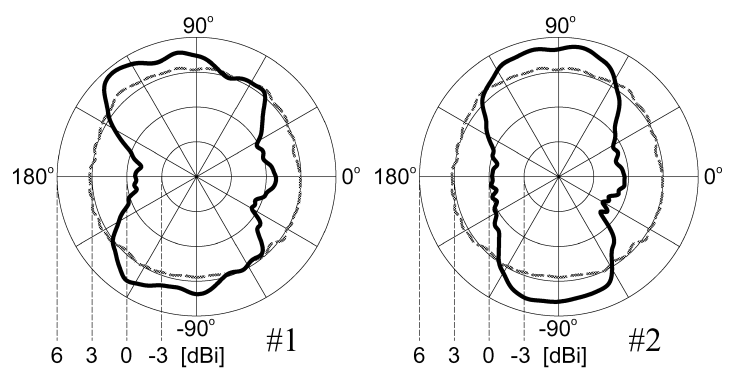

(a) $\mathrm{AS}=\lambda / 2$

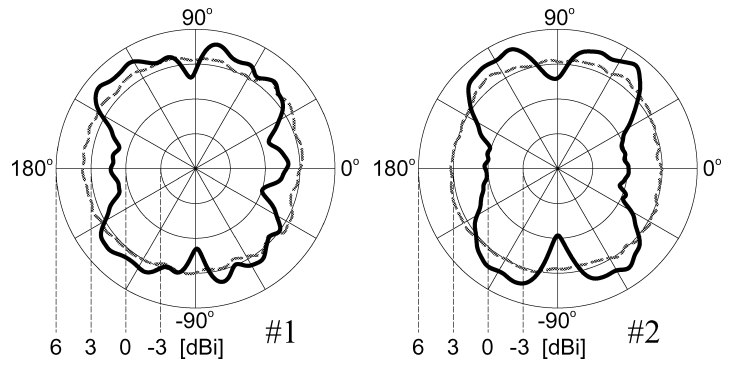

(b) AS $=1 \lambda$

Fig. 6 Antenna patterns for a single antenna (dashed) and for each fourelement ULA with mutual coupling (solid), extracting antennas \#1 (outer) and \#2 (inner).

tion. Focusing on the case of TX- $y / \mathrm{RX}-y$ with $\mathrm{AS}=\lambda / 2$ in $2 \times 2 \mathrm{MIMO}$, its gradient is clearly gentler compared with the other cases. This implies that relatively lower spacefrequency diversity gain was obtained in the case because the equivalently enlarged LOS component caused higher fading correlations and lower frequency selectivity. However, its performance is still better than that of the NLOS cases at a BER of $10^{-5}$. Since the estimated $K$-factor was $1.66 \mathrm{~dB}$, the total received power in the LOS environment should be $3.9 \mathrm{~dB}$ higher than that in the NLOS environment, theoretically. We confirmed that the actual received power in this LOS case was about $9 \mathrm{~dB}$ higher than that in the NLOS case due to the high antenna gain and other scattered waves. Despite the high received power, however, the uncoded performance in the LOS case does not show such gain compared with the NLOS case. This is due to high fading correlations mentioned above, which result in performance deterioration, especially in inverse matrix calculation in linear demultiplexing. On the other hand, the other LOS cases have lower correlations whereas the LOS component is considered to decrease due to lower antenna gain in the LOS direction. As a result, the uncoded performance in any LOS cases obtains 3-5 dB gain compared with that in the corresponding NLOS case as shown in Figs. 3 and 4. We can say that, therefore, the gain in the LOS condition still yielded the outperformance even in the coded case, especially in the array configuration of $\mathrm{TX}-y / \mathrm{RX}-y$ with $\mathrm{AS}=\lambda / 2$ in $2 \times 2$ MIMO.

Although similar diversity gain loss is observed in the case of TX- $y / \mathrm{RX}-y$ with AS $=\lambda / 2$ in $4 \times 4$ MIMO, it does not appear evident compared with the case in $2 \times 2 \mathrm{MIMO}$. It is supposed that a MIMO system equipped with more anten- nas can obtain higher spatial diversity effect even in a highly correlated case. From these results, we can say that a cyclic delay diversity technique [3], [11] is effective for solving the low-diversity issue in LOS environments, especially for a system equipped with fewer antennas such as $2 \times 2$ MIMO. It should be noted that, however, we could hardly see the low diversity gain in the LOS case when using maximum likelihood detection (MLD) at the receiver while we omit to show its performance. Therefore, we can say that the decrease in space-frequency diversity gain in the LOS case is a specific phenomenon when using a linear demultiplexing scheme such as MMSE, and that nonlinear detection algorithms, e.g., MLD, may not cause such a phenomenon.

We notice that the BER curves in the NLOS TX- $y / \mathrm{RX}$ $y$ scenario match well regardless of the AS. From Figs. 5 and 6 , we can see that the antenna patterns in the case of $\mathrm{AS}=\lambda / 2$ are similar to those in the case of AS $=1 \lambda$ except the regions around $90^{\circ} /-90^{\circ}$ directions. As aforementioned, the antenna gain in the $90^{\circ}$ direction, which corresponds to the LOS direction in the TX-y/RX- $y$ orientation, affects the performance in the LOS condition. In the NLOS condition, however, we can consider that the gain in the direction has little impact on the performance because the direct wave is obstructed. Therefore, it is considered that, in the NLOS condition, amplitudes of scattered waves observed by antenna arrays with mutual coupling were similar between the two cases of AS $=\lambda / 2$ and $1 \lambda$ in the TX- $y / \mathrm{RX}-y$ orientation. As proof of that, from CDFs of channel amplitudes and eigenvalue distributions shown in [8] and [9], respectively, we can see that channel gains in the two cases are similar. Although the fading correlations in the case of AS $=\lambda / 2$ are higher than those in the case of AS $=1 \lambda$ due to narrower AS, they are still less than 0.3 and are considered not to deteriorate the performance. Consequently, the agreement in the performance between the two AS cases seems to be due to the similarity of the propagation environments observed by antenna arrays.

\section{Conclusions}

By using channel data measured in an indoor environment, we have examined the practical performance of MIMOOFDM spatial multiplexing. It has been shown that in a coded case the LOS condition still provides better performance than the NLOS condition in the measurement site because of the benefit from higher received power yielded by the direct wave. However, it should be noted that MIMOOFDM spatial multiplexing systems in LOS environments may obtain lower space-frequency diversity effect when using a linear demultiplexing scheme.

\section{Acknowledgement}

This work was supported in part by a Research Fellowship for Young Scientists from the Japan Society for the Promotion of Science. 


\section{References}

[1] D. Gesbert, M. Shafi, D.S. Shiu, P. Smith, and A. Naguib, "From theory to practice: An overview of MIMO space-time coded wireless systems," IEEE J. Sel. Areas Commun., vol.21, no.2, pp.281-302, April 2003.

[2] E. Biglieri, R. Calderbank, A. Constantinides, A. Goldsmith, A. Paulraj, and H.V. Poor, MIMO Wireless Communications, Cambridge University Press, New York, 2007.

[3] IEEE802.11, The Working Group Setting the Standards for Wireless LANs, http://www.ieee802.org/11/

[4] S. Nanda, R. Walton, J. Ketchum, M. Wallace, and S. Howard, "A high-performance MIMO OFDM wireless LAN," IEEE Commun. Mag., vol.43, no.2, pp.101-109, Feb. 2005.

[5] A. Ghosh, D.R. Wolter, J.G. Andrews, and R. Chen, "Broadband wireless access with WiMax/802.16: Current performance benchmarks and future potential," IEEE Commun. Mag., vol.43, no.2, pp.129-136, Feb. 2005.

[6] H. Nishimoto, Y. Ogawa, T. Nishimura, and T. Ohgane, "Availability of MIMO spatial multiplexing in line-of-sight channels," Proc. IEEE APWCS2005, pp.40-44, Aug. 2005.

[7] Y. Ogawa, H. Nishimoto, T. Nishimura, and T. Ohgane, "Performance of MIMO spatial multiplexing in indoor line-of-sight environments," Proc. IEEE VTC2005-Fall, vol.4, pp.2398-2402, Sept. 2005.

[8] H. Nishimoto, Y. Ogawa, T. Nishimura, and T. Ohgane, "Measurement-based performance evaluation of MIMO spatial multiplexing in a multipath-rich indoor environment," IEEE Trans. Antennas Propag., vol.55, no.12, pp.3677-3689, Dec. 2007.

[9] H. Nishimoto, Y. Ogawa, T. Nishimura, and T. Ohgane, "MIMO E-SDM transmission performance in an actual indoor environment," IEICE Trans. Commun., vol.E90-B, no.6, pp.1474-1486, June 2007.

[10] T. Ohgane, T. Nishimura, and Y. Ogawa, "Applications of space division multiplexing and those performance in a MIMO channel," IEICE Trans. Commun., vol.E88-B, no.5, pp.1843-1851, May 2005.

[11] S. Kaiser, "Spatial transmit diversity techniques for broadband OFDM systems," Proc. IEEE GLOBECOM 2000, vol.3, pp.1824 1828, Nov. 2000 